\title{
Editorial
}

\section{Predicting Nonsentinel Node Metastases in Sentinel Node-Positive Breast Cancer: What Have We Learned, Can We Do Better, and Do We Need To?}

\author{
Hiram S. Cody III, MD and Kimberly J. Van Zee, MD
}

Breast Service, Department of Surgery, Memorial Sloan-Kettering Cancer Center, 1275 York Avenue, New York, NY 10021, USA

Sentinel lymph node (SLN) biopsy has become standard care for lymph node staging in breast cancer, represents a substantial step in the evolution of breast cancer surgery toward greater conservatism, and is one of the great success stories in contemporary surgical oncology. The most salient surgical questions (feasibility, accuracy, case selection, technique, and morbidity) have been asked and answered, and it is increasingly difficult to generate debate on any of them. In contrast, most aspects of SLN pathology remain controversial and elude consensus. Is intraoperative assessment worthwhile? Which method (frozen section, touch prep or smear) is best? How should SLN be processed for permanent pathology [single-section hematoxylin and eosin (H\&E) and/or serial sections and/or immunohistochemistry (IHC)]? What is the prognostic significance of SLN micrometastases, especially those detected only by IHC, or as pNOi + disease $(\leq 0.2 \mathrm{~mm}$ in size)? Is completion axillary dissection (ALND) required for all patients with SLN who are positive on final pathology? Is there a low-risk group for whom ALND is unnecessary and can we reliably identify this group? All of these issues are highly interrelated, but the last remains the most perplexing for surgeons and is the subject of a substantial literature. What have we learned?

\section{PREDICTING METASTASIS TO NON-SLN}

Non-SLN metastases are present in $40-50 \%$ of SLN-positive patients ${ }^{1,2}$ and are predicted by the same variables which predict metastasis to the SLN (or to axillary nodes in general): the most important are tumor size and lymphovascular invasion (LVI). Non-SLN metastases are also predicted by the characteristics of the SLN metastasis: the most important are method of detection (frozen section, $\mathrm{H} \& \mathrm{E}$, serial sections, or IHC), size of SLN metastasis $[<0.2 \mathrm{~mm}$ $(\mathrm{pN} 0 \mathrm{i}+), 0.2-2 \mathrm{~mm}(\mathrm{pN} 1 \mathrm{mi}),>2 \mathrm{~mm}(\mathrm{pN} 1)]$, number of positive $S L N$, presence of extranodal invasion, and number of negative SLN removed. Many papers predict non-SLN metastases on the basis of one or more variables, as previously summarized by Van Zee (20 studies in 26-702 patients). ${ }^{3}$ A meta-analysis by Degnim et al. ${ }^{4}$ (11 studies in 60-389 patients) found that non-SLN metastasis was most strongly associated with tumor size, LVI, more than one positive SLN, SLN metastasis $>2 \mathrm{~mm}$, and extranodal extension. A meta-analysis by Cserni et al. ${ }^{5}$ (25 studies) found that non-SLN metastases were present in $20 \%$ of patients with low-volume SLN disease and in $9 \%$ with SLN metastases detected only by IHC. Finally, a large series by Viale et al. ${ }^{6}$ of 1,228 SLNpositive patients found that the risk of non-SLN metastasis in patients with the most favorable combination of predictive factors was no less than $13 \%$.

\section{THE MSKCC NOMOGRAM}

Published online August 29, 2008.
Address correspondence and reprint requests to: Hiram S. Cody III, MD; E-mail: codyh@mskcc.org

Published by Springer Science+Business Media, LLC $\odot 2008$ The Society of Surgical Oncology, Inc.

\section{THE MSKCC NOMOGRAM}

The prediction of non-SLN status on the basis of one or a few variables is problematic, with risk 
estimates that vary widely between studies. The most logical response is to develop a multivariate model from a large dataset, and to validate it in a separate cohort of patients. Van Zee et al. ${ }^{7}$ have done so, drawing on our own experience in 1,075 SLN-positive patients who had a completion ALND. Multivariate logistic regression was used in 702 patients to develop a multivariate nomogram, the Memorial Sloan-Kettering Cancer Center (MSKCC) nomogram. The eight variables include tumor size, type/grade, LVI, multifocality, estrogen receptor (ER) status, method of SLN metastasis detection, number of SLN positive, and number of SLN negative. This model was validated prospectively in another 373 patients, and in a calibration plot there was good agreement across a wide range of probabilities between the predicted and the observed rates of non-SLN metastasis. The MSKCC nomogram (along with a more recent nomogram for the prediction of SLN metastasis ${ }^{8}$ ) is available online (www.mskcc.org/nomograms) in the form of a simple calculator.

\section{VALIDATING THE MSKCC NOMOGRAM}

The MSKCC nomogram is simply a test to predict the probability of non-SLN metastasis. The most direct validation is to compare the predicted and observed rates of non-SLN metastasis; in our own calibration plots, the nomogram performs well across a broad range of predicted rates. ${ }^{7}$ A more comprehensive measure of its performance is the receiver operating characteristic (ROC) curve. ROC curves were developed in World War II as part of a field called signal detection theory, and were used to evaluate the ability of radar operators to distinguish between the signals of enemy and friendly ships. They are now widely used in medicine to assess test performance; we recommend a particularly lucid online discussion of this topic by Tape. ${ }^{9}$

The ROC curve plots true positives (sensitivity) on the $\mathrm{Y}$ axis against false positives $(1-$ specificity) on the $\mathrm{X}$ axis (Fig. 1). A test's accuracy (ability to distinguish between patients with and without a condition) is represented by the area under the curve (AUC). An AUC of 1 (a vertical line along the $Y$ axis) indicates a perfect test with no false-positive results; an AUC of 0.5 (a diagonal line at $45^{\circ}$ ) indicates a worthless test with an equal number of true-positive and false-positive results, the equivalent of a coin toss.

Most diagnostic tests, including our nomogram, are imperfect and have an AUC somewhere in be-

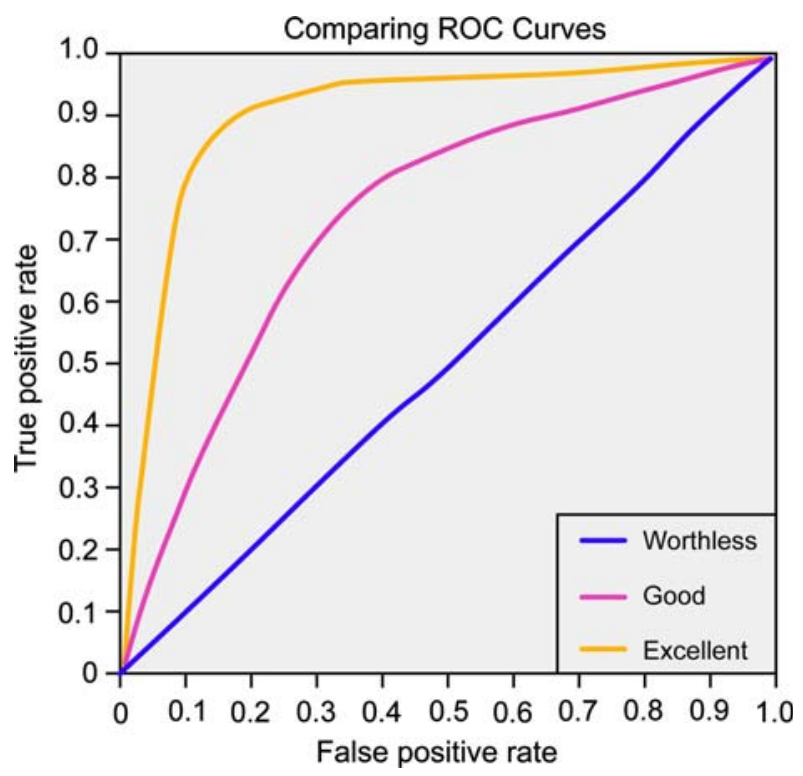

FIG. 1. A comparison of ROC curves for excellent, good, and worthless diagnostic tests. In a worthless test $(\mathrm{AUC}=0.50)$, there are equal numbers of true-positive and false-positive results; adapted from Tape. ${ }^{9}$

tween. The MSKCC nomogram AUC of 0.77 means that, between two randomly selected SLN-positive patients of whom one has a positive non-SLN, the nomogram would correctly identify that patient $77 \%$ of the time. The MSKCC nomogram has been validated by 15 studies worldwide (Table 1), ${ }^{7,10-22}$ including that of Poirier et al. ${ }^{22}$ in this issue of the Annals of Surgical Oncology, and other validation series are certain to follow. The nomogram had proved robust despite differences in patient demographics, clinical characteristics, surgical technique, and pathologic processing. The AUC values range from 0.58 to 0.86 and as one might expect, the highest $(0.82$ and 0.86$)$ and lowest $(0.58)$ values come from studies with fewer than 100 patients.

\section{CRITIQUING THE NOMOGRAM: CAN WE DO BETTER?}

Nomogram development lends itself to statistical tweaking, and many groups have raised caveats and proposed improved models. Degnim et al. ${ }^{11}$ selected subsets of their patients in whom the MSKCC nomogram predicted $\leq 5 \%$ and $\leq 10 \%$ probabilities of non-SLN metastases, and observed false-negative rates of $17 \%$ and $11 \%$, respectively; using a modified model, they were able to reduce the false-negative 
TABLE 1. Results of series validating the MSKCC nomogram

\begin{tabular}{lcc}
\hline \multicolumn{1}{c}{ Reference } & Patients $(n)$ & AUC \\
\hline${\text { Van Zee et al. }(2003)^{7}}_{\text {Smidt et al. }(2005)^{10}}$ & 373 & 0.77 \\
Degnim et al. (2005, Mayo) $^{11}$ & 222 & 0.78 \\
Degnim et al. (2005, Michigan) $^{11}$ & 465 & 0.72 \\
Soni et al. (2005) $^{12}$ & 89 & 0.86 \\
${\text { Dauphine et al. }(2007)^{13}}_{\text {Cripe et al. }(2006)^{14}}$ & 149 & 0.75 \\
${\text { Lambert et al. }(2006)^{15}}_{\text {Zgajnar et al. }(2007)^{16}}$ & 51 & 0.63 \\
${\text { Alran et al. }(2007)^{17}}_{\text {Cserni (2007) }}^{18}$ & 92 & 0.82 \\
Pal et al. $(2008)^{19}$ & 200 & 0.71 \\
Klar et al. $(2008)^{20}$ & 276 & 0.72 \\
Kohrt et al. $(2008)^{21}$ & 588 & 0.72 \\
Poirier et al. $(2008)^{22}$ & 140 & 0.73 \\
& 118 & 0.68 \\
\end{tabular}

MSKCC, Memorial Sloan-Kettering Cancer Center; AUC, area under the ROC curve.

rate for low-risk patients, but without increasing the AUC. Alran et al. ${ }^{17}$ observed an AUC of 0.72 for all of their patients, but only 0.52 for patients with SLN micrometastases $(\leq 2 \mathrm{~mm})$, and concluded that the nomogram was not reliable in low-probability cases. Kohrt et al., ${ }^{21}$ using three different statistical techniques, developed a model based on three variables (tumor size, LVI, and SLN metastasis size) which outperformed the MSKCC nomogram in their own patients, with AUCs of $0.83-0.85$ versus 0.77 . Of note, only $60 \%$ of their 285 patients had complete pathologic data. Coutant et al. ${ }^{23}$ reported a scoring system based on three variables (tumor size, presence/ absence of macrometastasis, and no. of positive SLNs divided by no. of SLNs removed), and achieved an AUC of 0.82. Pal et al. ${ }^{19}$ added SLN metastasis size to their model and improved the AUC from 0.68 (MSKCC) to 0.84 . Finally, Dauphine et al. ${ }^{13}$ (in 39 patients) applied three different scoring systems, observed AUCs of $0.63,0.70$, and 0.68, and advised caution in the application of each.

It is worth emphasizing that subset analyses of nomogram performance are problematic. Alran et al. ${ }^{17}$ observed a poor AUC (0.52) for patients with SLN micrometastases, but their own unpublished data (S. Alran, personal communication) indicate good agreement between the observed and predicted rates of non-SLN involvement for patients with SLN micrometastases $\leq 2 \mathrm{~mm}$ (14\% observed versus $10 \%$ predicted) and for patients with SLN positive only on IHC ( $11 \%$ observed versus $9 \%$ predicted). In this issue of the Annals of Surgical Oncology, Poirier et al. ${ }^{22}$ report that, for their patients with nomogram scores of $\leq 10 \%$ ( $18 \%$ of all cases), the observed rate of non-SLN metastasis was $13 \%$ [95\% confidence interval (CI) 2-24\%]. Based on a small sample size $(n=37)$ with wide confidence intervals, this degree of variation is not at all surprising. Taken together, these studies demonstrate that: (1) a low AUC in a small subset indicates lack of precision in being able to discriminate between two numbers within a small range (for example $11 \%$ versus $9 \%$ ), a deficiency which is not clinically relevant, and (2) an observed rate of non-SLN metastasis lower or higher than the predicted value neither proves nor disproves the value of the nomogram. In fact, the nomogram performed well in both studies.

There is to date no standardized methodology for pathologic analysis of SLN, and this inconsistency may account for at least some of the observed variation in nomogram performance. We began to perform SLN biopsy in 1996, adopted a pathologic protocol using serial sections/IHC, ${ }^{24}$ and, in the MSKCC nomogram, categorized SLN metastasis by pathologic detection (frozen section, routine $\mathrm{H} \& \mathrm{E}$, serial sections, IHC-only). Current American Joint Committee on Cancer (AJCC) staging ${ }^{25}$ categorizes nodal metastases on the basis of size $(\leq 0.2 \mathrm{~mm}, 0.2$ $2 \mathrm{~mm},>2 \mathrm{~mm}$ ) and we are now updating the nomogram on this basis. We agree with Turner et al. ${ }^{26}$ that AJCC lymph-node staging is subject to wide interpretive variation, and that there is substantial room for improvement. Lymph node staging should be simple, reproducible, cost effective, and clinically relevant; in current practice, we have not yet achieved these goals.

\section{THE NOMOGRAM IN EVERYDAY PRACTICE: WHAT IT CAN AND CANNOT DO}

First, the MSKCC nomogram was designed to estimate the probability of non-SLN metastases in SLN-positive patients, not to determine with certainty that non-SLN disease is (or is not) present. It is crucial to recognize that the nomogram correctly discriminates between randomly selected patients with and without SLN metastases in about threequarters of cases, i.e., that it is not perfect.

Second, the MSKCC nomogram is superior to clinical judgment. In two separate studies using hypothetical scenarios, ${ }^{27,28}$ the nomogram outperformed clinician "guesstimates" (in one of these, ${ }^{27}$ the AUC values for nomogram versus clinicians were 0.72 versus $0.54, P<0.01)$. It is again crucial to recognize that the nomogram is a more accurate guess than clinical judgment, but that it remains a guess. 
Third, the MSKCC nomogram cannot tell us (or our patients) what to do; there is no cutoff nomogram score which mandates the performance of an ALND. When Poirier et al. ${ }^{22}$ in this issue of the Annals of Surgical Oncology state that 71\% of Quebec surgeons would not perform an ALND for nomogram scores of $\leq 10 \%$, they imply a $10 \%$ cutoff. While we have observed a declining rate of ALND in our SLN-positive patients and lower nomogram scores in SLNpositive patients who did not have ALND compared with those who did $(10 \%$ versus $37 \%)$, the range in nomogram scores for the no-ALND patients was wide (1-89). ${ }^{29}$ The decision for ALND in SLN-positive patients should be individualized considering multiple factors (patient age, comorbidities, anxiety level, and implications for systemic therapy, among others), and not based on the nomogram score alone.

\section{FUTURE DIRECTIONS: ARE WE ASKING THE WRONG QUESTION?}

At present, the most important reasons for ALND in SLN-positive patients are to guide systemic therapy and to prevent local recurrence. The decision for systemic therapy is multifactorial, and, for SLN-positive patients, is infrequently changed by the discovery of additional positive nodes. For the occasional SLNpositive patient in whom systemic therapy might be changed, completion ALND is reasonable. In our opinion, this assessment is better made by the medical oncologist than the surgeon.

Regarding local control, six series $(n=583,2003-$ 2007) of selected SLN-positive/no-ALND patients report axillary local recurrence (LR) of $0.5 \%$ at a median follow-up of 31 months, results quite comparable to those in 14 series $(n=3802,2004-2007)$ of SLN-negative/no-ALND patients: $0.3 \%$ at 47 months' follow-up. ${ }^{30}$ In our own series of SLNpositive/no-ALND patients, ${ }^{29}$ we observed axillary LR as a first event in $1 \%$ ( 3 of 287 patients) at followup of 23 months. It seems inconceivable that these very low rates of axillary LR would ever reach $10 \%$, the level at which LR had a detectable adverse effect on survival in the most recent Early Breast Cancer Trialists' Collaborative Group overview. ${ }^{31}$ Across the burgeoning literature validating the MSKCC nomogram, we are in effect asking the question, "Which SLN-positive patients do not need ALND?" It is quite clear from the data above that at least some do not and that practice patterns are changing. The American College of Surgeons Oncology Group (ACOSOG) Z0011 trial ${ }^{32}$ (a randomization of SLN-positive patients to ALND versus observation) closed early due to slow accrual and low event rates, but was ahead of its time in asking a better question: "Which SLN-positive patients, if any, need ALND?" It is time to ask this question again.

\section{REFERENCES}

1. Kim T, Giuliano AE, Lyman GH. Lymphatic mapping and sentinel lymph node biopsy in early-stage breast carcinoma. Cancer 2006; 106:4-16

2. Krag DN, Anderson SJ, Julian TB, et al. Technical outcomes of sentinel-lymph-node resection and conventional axillarylymph-node dissection in patients with clinically node-negative breast cancer: results from the NSABP B-32 randomised phase III trial. Lancet Oncol 2007; 8:881-8.

3. Van Zee KJ. Residual disease in the axilla: to predict or not predict? Breast Diseases 2004; 15:231-4.

4. Degnim AC, Griffith KA, Sabel MS, et al. Clinicopathologic features of metastasis in nonsentinel lymph nodes of breast carcinoma patients. Cancer 2003; 98:2307-15.

5. Cserni G, Gregori D, Merletti F, et al. Meta-analysis of nonsentinel node metastases associated with micrometastatic sentinel nodes in breast cancer. Br J Surg 2004; 91:1245-52.

6. Viale G, Maiorano E, Pruneri G, et al. Predicting the risk for additional axillary metastases in patients with breast carcinoma and positive sentinel lymph node biopsy. Ann Surg 2005; 241:319-25.

7. Van Zee KJ, Manasseh DM, Bevilacqua JL, et al. A nomogram for predicting the likelihood of additional nodal metastases in breast cancer patients with a positive sentinel node biopsy. Ann Surg Oncol 2003; 10:1140-51.

8. Bevilacqua JL, Kattan MW, Fey JV, et al. Doctor, what are my chances of having a positive sentinel node? A validated nomogram for risk estimation. J Clin Oncol 2007; 25:3670-9.

9. Tape TG. The area under an ROC curve, 2008. Available from: http://gim.unmc.edu/dxtests/roc3.htm. Accessed August $25,2008$.

10. Smidt ML, Kuster DM, van der Wilt GJ, et al. Can the Memorial Sloan-Kettering Cancer Center nomogram predict the likelihood of nonsentinel lymph node metastases in breast cancer patients in the Netherlands? Ann Surg Oncol 2005; 12:1066-72.

11. Degnim AC, Reynolds C, Pantvaidya G, et al. Nonsentinel node metastasis in breast cancer patients: assessment of an existing and a new predictive nomogram. Am J Surg 2005; 190:543-50.

12. Soni NK, Carmalt HL, Gillett DJ, et al. Evaluation of a breast cancer nomogram for prediction of non-sentinel lymph node positivity. Eur J Surg Oncol 2005; 31:958-64.

13. Dauphine CE, Haukoos JS, Vargas MP, et al. Evaluation of three scoring systems predicting non sentinel node metastasis in breast cancer patients with a positive sentinel node biopsy. Ann Surg Oncol 2007; 14:1014-9.

14. Cripe MH, Beran LC, Liang WC, et al. The likelihood of additional nodal disease following a positive sentinel lymph node biopsy in breast cancer patients: validation of a nomogram. Am J Surg 2006; 192:484-7.

15. Lambert LA, Ayers GD, Hwang RF, et al. Validation of a breast cancer nomogram for predicting nonsentinel lymph node metastases after a positive sentinel node biopsy. Ann Surg Oncol 2006; 13:310-20.

16. Zgajnar J, Perhavec A, Hocevar M, et al. Low performance of the MSKCC nomogram in preoperatively ultrasonically neg- 
ative axillary lymph node in breast cancer patients. $J$ Surg Oncol 2007; 96:547-53.

17. Alran S, De RY, Fourchotte V, et al. Validation and limitations of use of a breast cancer nomogram predicting the likelihood of non-sentinel node involvement after positive sentinel node biopsy. Ann Surg Oncol 2007; 14:2195-201.

18. Cserni G. Comparison of different validation studies on the use of the Memorial Sloan-Kettering Cancer Center nomogram predicting nonsentinel node involvement in sentinel node-positive breast cancer patients. Am J Surg 2007; 194:699-700.

19. Pal A, Provenzano E, Duffy SW, et al. A model for predicting non-sentinel lymph node metastatic disease when the sentinel lymph node is positive. $B r J$ Surg 2008; 95:302-9.

20. Klar M, Jochmann A, Foeldi M, et al. The MSKCC nomogram for prediction the likelihood of non-sentinel node involvement in a German breast cancer population. Breast Cancer Res Treat 2008 (Epub ahead of print).

21. Kohrt HE, Olshen RA, Bermas HR, et al. New models and online calculator for predicting non-sentinel lymph node status in sentinel lymph node positive breast cancer patients. $B M C$ Cancer 2008; 8:66.

22. Poirier E, Sideris L, Dube P, et al. Analysis of clinical applicability of the Breast Cancer Nomogram for positive sentinel lymph node: the Canadian Experience. Ann Surg Oncol 2008; 15:2562-7.

23. Coutant C, Rouzier R, Fondrinier E, et al. Validation of the Tenon breast cancer score for predicting non-sentinel lymph node status in breast cancer patients with sentinel lymph node metastasis: a prospective multicenter study. Breast Cancer Res Treat 2008 (Epub ahead of print).

24. Brogi E, Torres-Matundan E, Tan LK, et al. The results of frozen section, touch preparation, and cytological smear are comparable for intraoperative examination of sentinel lymph nodes: a study in 133 breast cancer patients. Ann Surg Oncol $2005 ; 12: 173-80$

25. American Joint Committee on Cancer. AJCC Cancer Staging Manual, 6th edition. New York: Springer-Verlag, 2002.

26. Turner RR, Weaver DL, Cserni G, et al. Nodal stage classification for breast carcinoma: improving interobserver reproducibility through standardized histologic criteria and imagebased training. J Clin Oncol 2008; 26:258-63.

27. Specht MC, Kattan MW, Gonen M, et al. Predicting nonsentinel node status after positive sentinel lymph biopsy for breast cancer: clinicians versus nomogram. Ann Surg Oncol 2005; 12:654-9.

28. Smidt ML, Strobbe LJ, Groenewoud HM, et al. Can surgical oncologists reliably predict the likelihood for non-SLN metastases in breast cancer patients? Ann Surg Oncol 2007; 14:615-20.

29. Park J, Fey JV, Naik AM, et al. A declining rate of completion axillary dissection in sentinel lymph node-positive breast cancer patients is associated with the use of a multivariate nomogram. Ann Surg 2007; 245:462-8.

30. Rutgers EJT. Sentinel node biopsy: interpretation and management of patients with immunohistochemistry-positive sentinel nodes and those with micrometastases. J Clin Oncol 2008; 26:698-702.

31. Early Breast Cancer Trialists' Collaborative Group. Effects of radiotherapy and of differences in the extent of surgery for early breast cancer on local recurrence and 15-year survival: an overview of the randomised trials. Lancet 2005;366:2087-106.

32. Giuliano AE. Z0011: a randomized trial of axillary node dissection in women with clinical T1 or T2 N0 M0 breast cancer who have a positive sentinel node; 2003. Available from http://www.acosog.org/studies/synopses/Z0011_Synopsis.pdf. Accessed August 25, 2008. 